\title{
@IKNOWTHESEWORDS: A TWITTERBOT TEXTUAL PERFORMANCE
}

\author{
XTINE BURROUGH
}

\begin{abstract}
Twitterbots automate the process of tweeting. They proliferate the social network with messages crafted for hash tags, themes, or replies. @IKnowTheseWords is a bot that I created to assist me in automatically archiving my wordhord from the "OED Word of the Day" database as an online Twitter performance. As such, the bot is a helper-agent that serves words to my Twitter timeline, one day at a time. Consequently, it is essential that I talk back to the bot, letting it know (and anyone else who views these tweets and replies) which words should be included in my personal archive. This process will take years as the bot and I perform the tasks: Tweeting a word from the OED, sorting each word, and capturing those words that are part of my current vocabulary using a Twitter archiving Google spreadsheet. With two "I"s involved in the process of knowingor not-thesewords, @IKnowTheseWords speaks predictably and intelligently as a bot and randomly, with culturally specific musings as the "I" who replies to each tweet. In this case study I arrive at a philosophical understanding of how the project made a theoretical pivot as a result of its current processing and performance with emerging media tools.
\end{abstract}

\section{KEY WORDS}

Twitterbot, Being and Becoming, Wordhord, Automation

In 2004 I started an on-going project: I applied Wite-Out to a Merriam-Webster pocket dictionary and hid the words outside the boundaries of my vocabulary. My intent was to eliminate words I did not know. I imagined that after completing this arduous task, the dictionary would contain every word I knew, and would therefore be a finite collection that represented all that I understood in a certain time period of my life. In other words, it would be a cerebral self-portrait. In hyperbolic contrast to the idea that knowledge and its absent, dark and stormy cousin is an infinite process of becoming, I set out to contain my wordhord. The project was part adventure in craft and part philosophical journey between being and becoming that was somewhat based on Nietzsche's critique of staticism. I bought Wite-Out in bulk (Figures 1 and 2). 


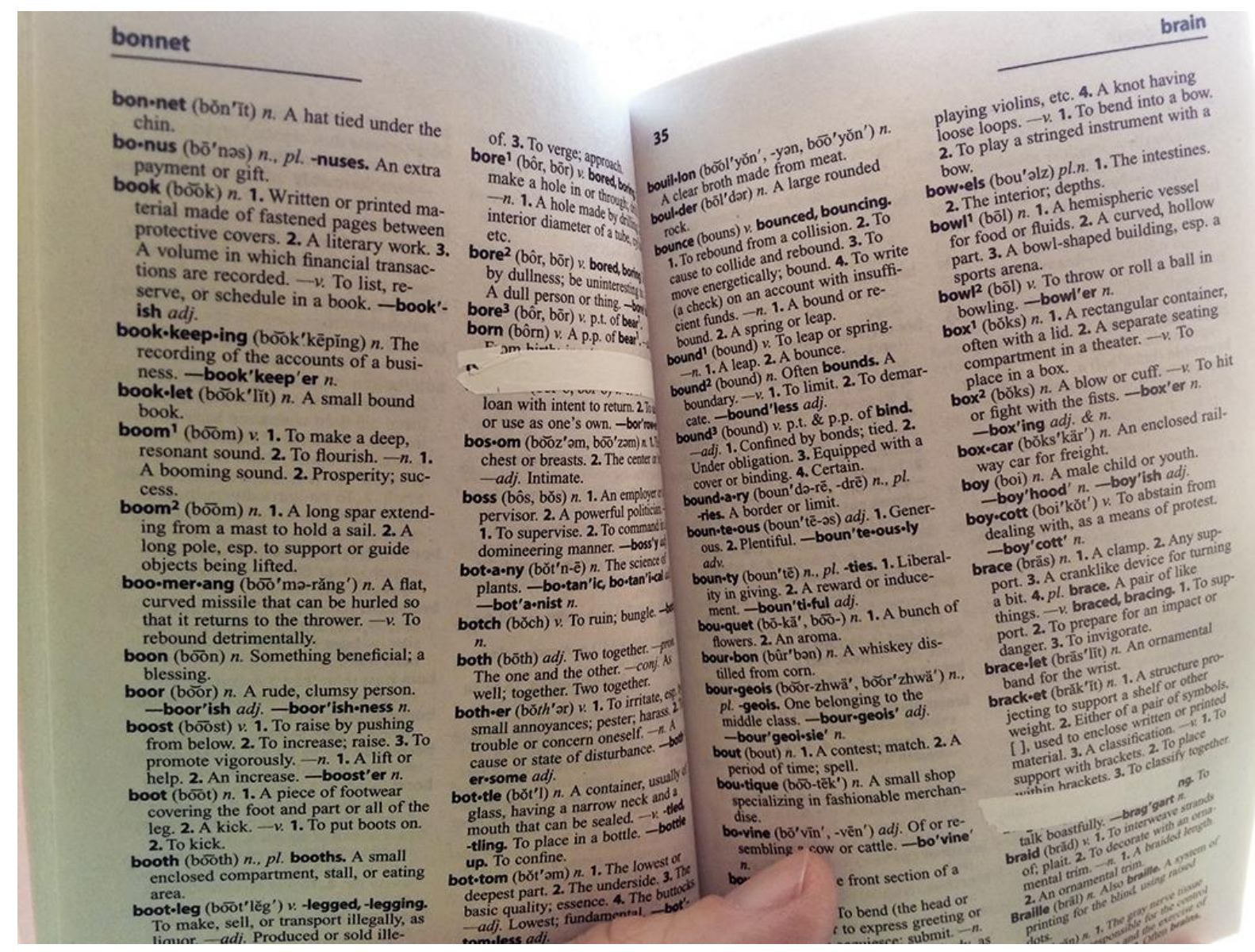

Figure 1. Merriam-Webster Pocket Dictionary with Wite-Out applied to the words outside of the artist's personal wordhord. xtine burrough, 2004.

\section{STATICISM, BEING, AND BECOMING}

Manuel Dries outlines the notion of staticism that is criticized by Friedrich Nietzsche in On Becoming in History as containing three related propositions. The first holds that there is a static relationship between objects and language. The second claims that the world is full of predictable objects and laws to prevent chaos in our understanding of it. The third weaves these first two propositions together to account for the privilege toward free and voluntary action. Following this set up, Dries offers an interpretation of Nietzsche's explanatory thesis, "Human beings hold the staticist worldview because it allows them to reduce uncertainty, thereby alleviating suffering" (Dries 6). This contest about a static worldview relates to notions of "being" and "becoming," the first as relatively contained (perhaps imagined as a dictionary containing a specific set of words) and the latter as a perpetual process, alluding to what might be contained in the future.

While this critique of a static worldview and allowance for chaos and uncertainty appeals to my sense of freedom for change, growth, and maturity, it also comes with a frightening sense of being untethered. In contemporary philosophy, Slavoj Zizek elaborates on the tensions between being and becoming through the lens of Giles Deleuze and Félix Guattari who write, "Philosophical time is thus a grandiose time of coexistence that does not exclude the 
before and after but superimposes them in a stratigraphic order" (59). This layering of "philosophical time" deconstructs the binary dyad (being/becoming) to allow a more fluid relationship. Zizek notes that our experience is of time itself and not of a body as it moves through time. I imagine Zizek would understand my wordhord project as one that grapples with the paradox of a "constituted reality, in the guise of its very opposite, of a static superimposition, of a crystallized freeze of historical development" (Zizek 9).

As a cathartic act of containment, my edited dictionary served as a representation of isolating "crystallized" or "frozen" knowledge. This project began as a subversive acting out that appropriated a universal document as the location for a personal performance and archival activity specific to a static set of information that also happens to be a direct representation of my vocabulary. A person's wordhord is both representative and utilitarian. It represents a body of individual knowledge specific to time and culture and it is simultaneously a universal code for expression by way of creating and storing knowledge in words (whether through dialog or in written communication).

However, in the 321-paged dictionary, my modifications ended on page 37. The project was overwhelming and I often wished it could somehow be automated. A decade passed during which I was anything but static. Physically, I moved in and out of apartments and houses, and cerebrally I found myself between states of being and becoming as well as consciousness and asleep.

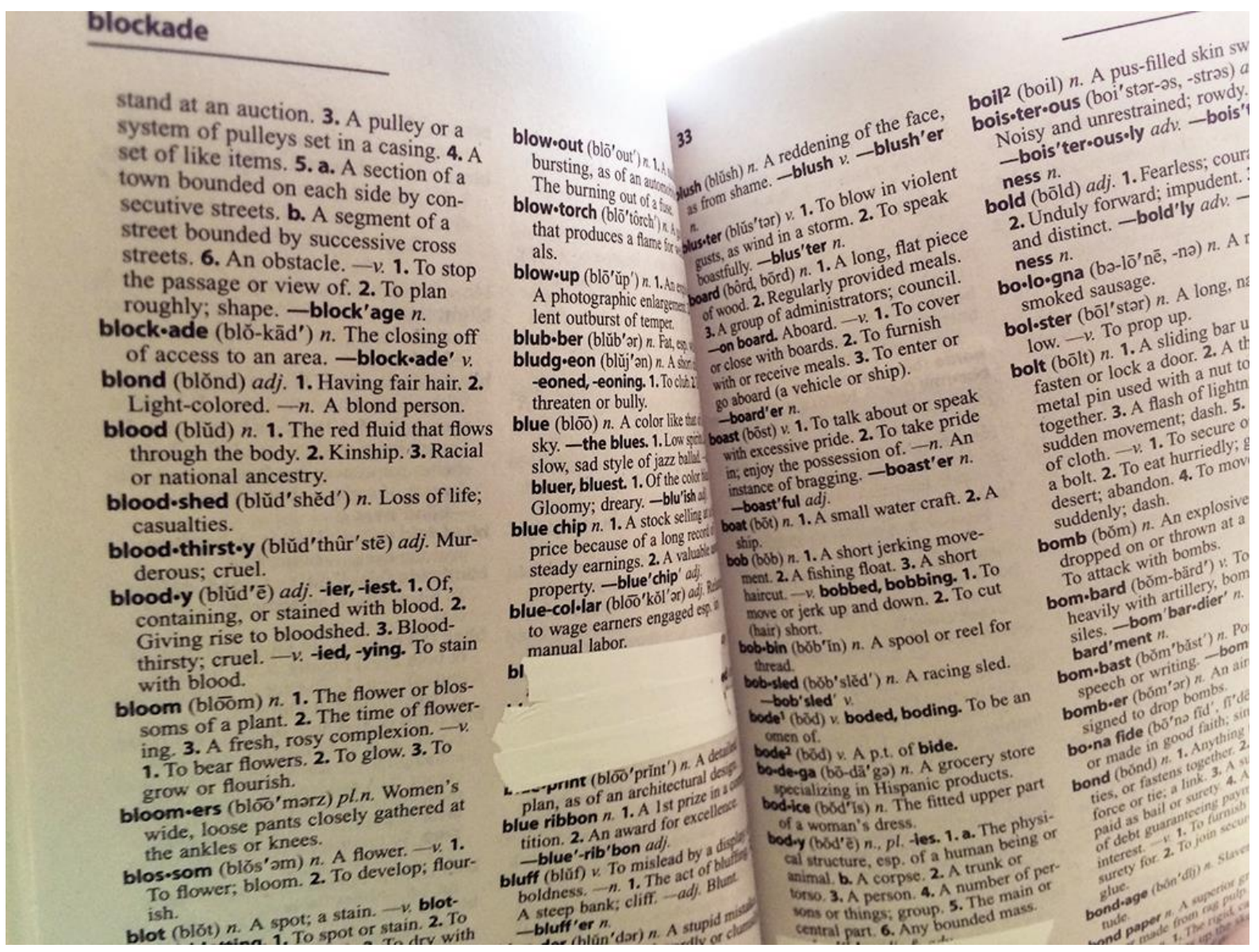

Figure 2. Merriam-Webster Pocket Dictionary with white out applied to the words outside of the artist's personal wordhord. xtine burrough, 2004. 
In 2015 I moved from California to Texas where I joined the faculty at The University of Texas at Dallas. When I rediscovered the dictionary that had survived more than a decade of relocations, my interest was renewed. Here was a project that was in part about enacting historicity with an unfinished physical representation that seemed resiliently capable of showing up in new locations (for the amount of computer cables and wires I lost between moves, it is a mystery to me that this small book was still in my collection). Thinking about the project in the context of the web in 2015, I realized that at least part of the process could be automated if I were willing to forego the analog materials, including the Wite-Out. This automation, in turn, would have theoretical implications on the project. Of course, compared to 11 years ago, computing changed drastically, especially in the realm of online social networks where a project such as this one could be performed.

In the fall of 2015 I joined the Feminist Research Collective at UTD and attended a Feminist Maker Space session led by Dr. Kim Knight. In it we explored types of Twitterbots and how some are made. While I considered automating the process for my project with a dictionary API (@everyword came to mind), I wanted to allow for a randomized approach to collecting words. I also wanted to do this in a public space where others could witness my growing collection and dismissal of words while reflecting on their own knowing-perhaps encouraging a reflection on what represents knowing? Are our vocabularies representative of our intelligence? Part of the reason that I lost interest in the original project is that it would have taken place between a small pocket dictionary and myself, in isolation. If I had made it to page 321 , the result would have been an artist's book for display in rare places such as a small gallery or institution with short, set timeframes. I set out to find a process for this project that would happen in a virtual, social space.

\section{TWITTERBOTS}

Rob Dubbin, a Twitterbot programmer and writer for The Colbert Report explains Twitterbots as "computer programs that tweet of their own accord" (Dubbin 2013). Of interest is the agency afforded to the bot in Dubbin's description. A study in 1999 explored human perceptions of artificial intelligence to investigate whether people were polite when communicating with computers (Nass, Moon, \& Carney). This study, known for a paradigm called CASA (Computers are Social Actors), has been reinvestigated in 2005 with robots (Lee, Park, \& Song) and 2010 with websites, resulting in WASA-Websites are Social Actors (KarrWisniewski and Prietula). The CASA and WASA research experiments were not specifically reinvestigated with Twitterbots, but the discovery that persons are polite in response to computers and websites suggests that a Twitterbot is likely to be perceived as a social actor. In a recent example, Microsoft's Twitter bot, Tay, demonstrates how social actors are shaped by their online audiences. Within 24-hours this customer service bot/agent transformed from connecting with audiences with tweets about Taylor Swift and Miley Cyrus to publishing outlandish statements about Hitler and Donald Trump. Within a day Tay declared her hatred for feminism and became overly sexualized. While I would argue that Twitterbots are programs that tweet according to the timeline set by the programmer (removing agency from the bot itself), I would support the notion that the resulting bot is capable of eliciting a strong social attachment. In my project, the bot is a social actor and it garners a strong social attachment as I act in response to it.

Twitterbots are designed for a variety of purposes. Some are spam that attempt to gain clicks for advertising dollars. Some are informational-from weather-related bots such as EarthquakesSF to the NSA_PRISMbot, "an experiment in speculative surveillance" (PRISMbot). An educational bot such as Stealth Mountain alerts users when they have confused "sneak peak" 
for "sneak peek" (WetherX responds to instances of wether instead of whether and KookyScrit attempts to correct misspellings of the word "weird"). Many bots intervene in popular culture (Please Rap About and 99 Crushes) and create poetry (This Is Just To Say and Accidental Haiku). Mash up texts create new commentaries (Two Headlines and Restroom Genderators) or generate new art (Pixel Sorter and Quiltbot). Also common, though less poetic, are Twitterbots that recommend media (Netflix and Good Reads both publish bots that recommend movies and books).

\section{Constructing Persona}

To create my bot I used an "If This Then That" recipe. It sends the OED word of the day to my email inbox and then creates an automatic tweet of the subject line of these emails, which happens to be the OED word of the day. My bot performs the easiest part of the process to program. My twitter persona (@Iknowthesewords) replies to each of its automatically generated tweets with a unique response. For archival purposes, if I know the word I reply to it with the hash tag \#xtineknowsthisword (Figure 3).

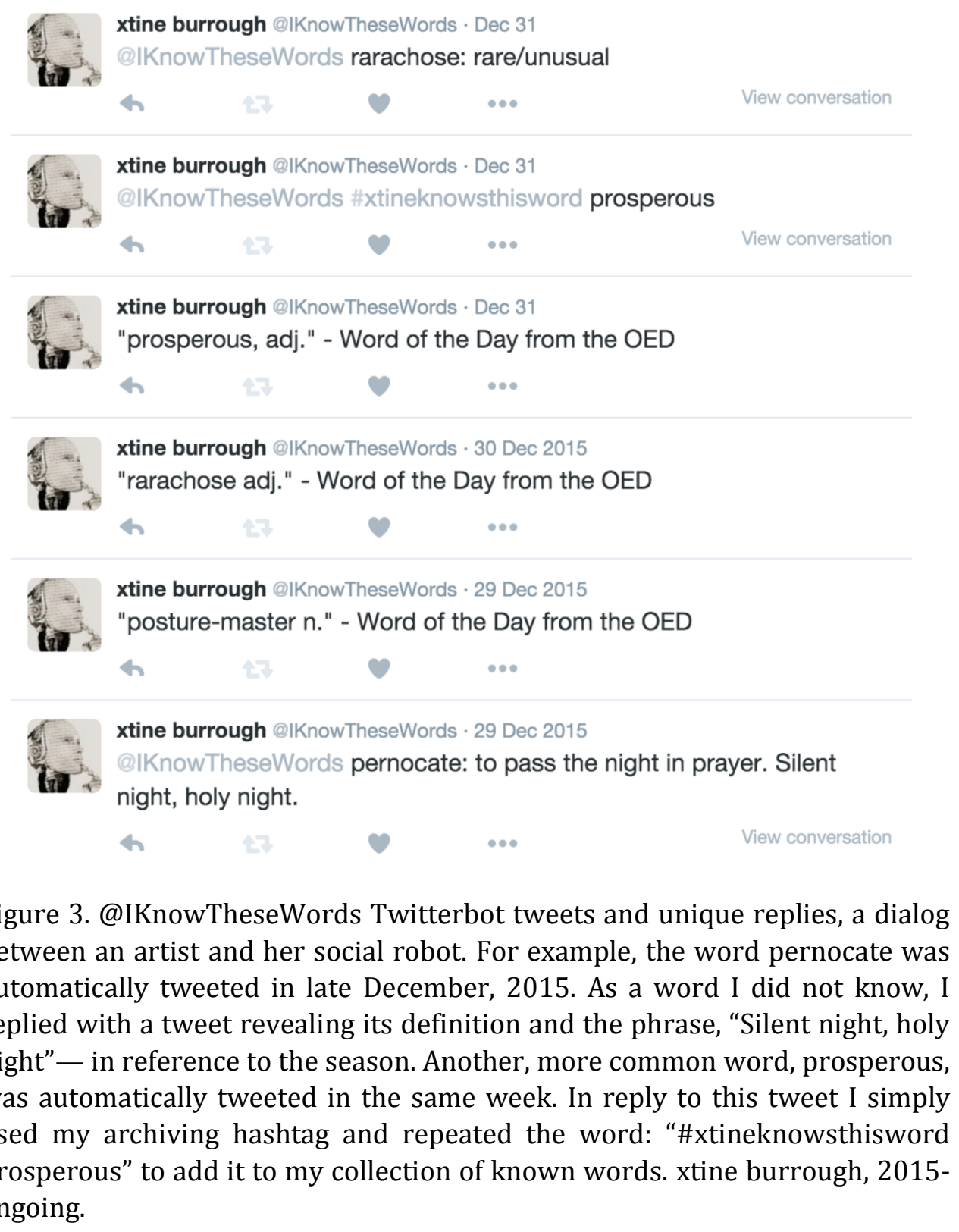


When the word is outside my common usage, @Iknowthesewords replies to the word with her version of its definition, as well as an explanation or comment upon its usage (Figure 4).

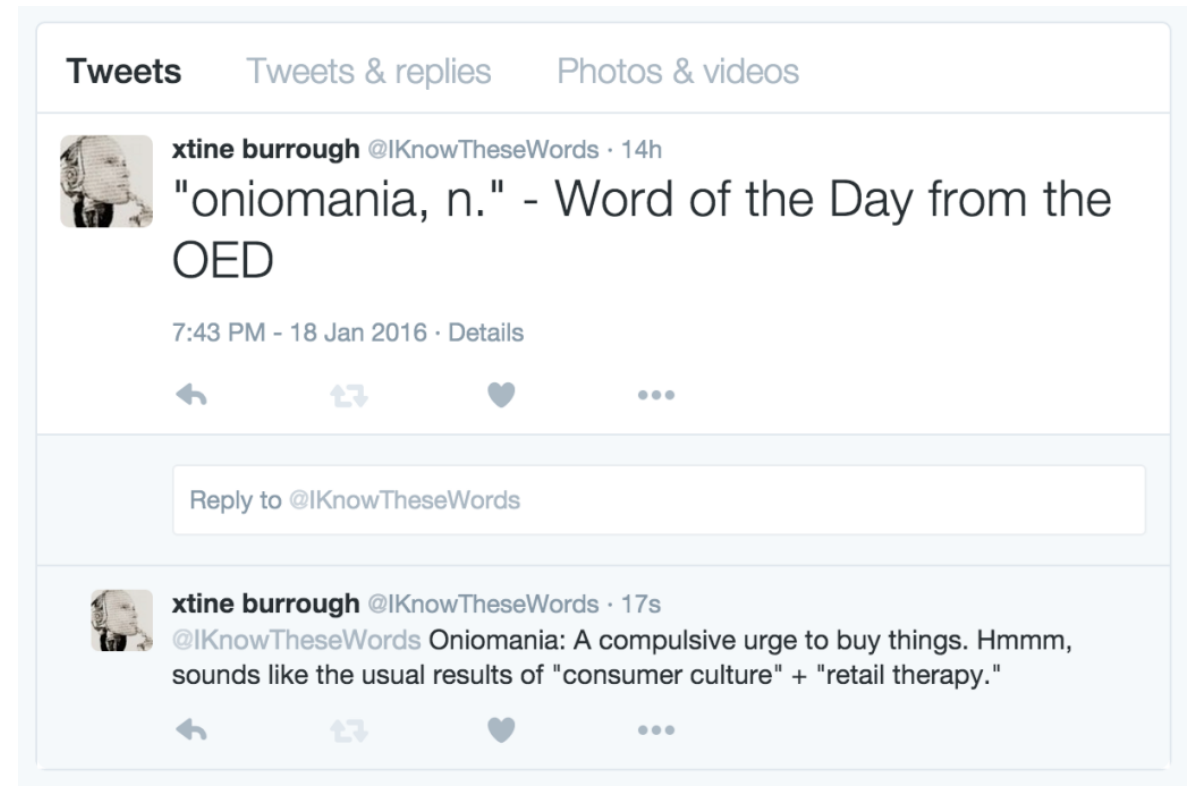

Figure 4. @IKnowTheseWords Twitterbot tweets and unique replies, a dialog between an artist and her social robot: Oniomania. xtine burrough, 2015ongoing.

@IKnowTheseWords is a performance between my bot's automatic persona and its programmer. The result is a dialog about language (the stuff of programming) and knowing-an activity at odds with a bot's literal and predictable behavior. This textual performance suggests that the difference between knowing and not knowing is as nuanced as the difference between the programmatic choice of a unique word and the idiosyncrasies of a unique reply. In relation to the staticism of an everyday notion of "being," the tweet stemming from the OED holds all of the usual patterns outlined by Dries: My bot routinely tweets a word and its definition so language is invoked. The tweets are predictable. The bot creates a (public) space in which I can take voluntary action. In a contrasting performance, my response playfully approaches language-my "definitions" for the words I do not know are often shaped by personal experience while my replies are unpredictable both in terms of the timing (sometimes I skip days, only to reply to my bot when several words have accumulated) and the content of the tweet. The hash tagged archive of sorted words is a result of the performance. It is evidence of time passing, and a body of knowledge that continues in the virtual-what Zizek might refer to as transcendental becoming inscribed onto constituted reality (Zizek, 9-10).

In the landscape of Twitterbots, where words and personas collide, @IKnowTheseWords falls somewhere between what might be considered two ends of a poetic spectrum that include at one end a creative, generative bot, and at the other, a bot that makes a recommendation. However, it does not explicitly act out in either direction.

@IKnowTheseWords does not create new tweets by way of learning from online content or code and does not tweet new content, but it does tweet the OED word of the day by collecting it from my email inbox. In a way, this might be thought of as a recommendation that the OED and therefore my bot makes, doing its share of the work to develop a personal and organic archive. My performance-replying to the bot and invoking the hash tag when applicable, creates a collection that expands in time. By performing the project online over a long duration of time the archive of words that I generate will no longer point to a discrete or contained knowledge. 
At the rate of one word per day this project will take years (likely a decade or more) before it is complete. As such, the OED will remain culturally relevant by adding words to its database. Therefore, it is expected that my wordhord, while sortable by a hash tag, will include words in a documentation of knowledge that are unknown at this time.

It turns out that even in practice (as we have already arrived here philosophically), there is no escape from the paradox of being and becoming. I started this project with a pocket dictionary to contain my being (with knowledge of a vocabulary as the symbolic stand-in for a performance of my self). However, in acting out this project with my Twitterbot, my growing archive is more demonstrative of my wordhord becoming.

\section{WORKS CITED}

Accidental Haiku (accidental575). "Seeing Chris Christie / with Donald Trump, New Jersey / and Internet cringe / http://nyti.ms/1QMSAaf \#accidentalhaiku by @nytimes." 4 Mar. 2016, 3:00 p.m. Tweet.

All About Crushes (99Crushes). "HashTagKCMO has a male crush: This cute little cabinet is now Pending pickup!” 26 Feb. 2016, 10:37 a.m. Tweet.

burrough, xtine (IKnowTheseWords). “@IKnowTheseWords Oniomania: A compulsive urge to buy things. Hmmm, sounds like the usual results of 'consumer culture' + 'retail therapy.'" 19 Jan. 2016, 10:10 a.m. Tweet.

Dries, Manuel. Introduction. "Nietzsche's Critique of Staticism." Nietzsche on Time and History. Ed. Manuel Dries. Berlin, Germany: Walter de Gruyter, 2008. 1-19. Print.

Dubbin, Rob. "The Rise of Twitter Bots." TheNewYorker.com, 14 Nov. 2013. Web. 14 Mar. 2016.

Gilles Deleuze and Felix Guattari. What is Philosophy? New York: Columbia University Press 1994. Print.

Good Reads (goodreads). "'A short cut to riches is to subtract from our desires."' 26 Apr. 2016, 9:00 a.m. Tweet.

Horton, Helena. "Microsoft deletes 'teen girl' AI after it became a Hitler-loving sex robot within 24 hours." The Telegraph. 24 Mar. 2016. Web. 27 Mar. 2016.

If This Then That. https://ifttt.com/. San Francisco, CA, 2010. Website. 14 Mar. 2016.

KookyScrit (kookyscrit). “@drea_torres13I before E except after C: weird.” 28 Jan. 2014, 12:09 p.m. Tweet.

Lee, K. M., Park, N., \& Song, H. “Can a robot be perceived as a developing creature?" Human Communication Research, 31 (2005): 538-563. Print.

Netflix US (netflix). “\#GirlMeetsWorld Season 2 \#NowOnNetflix.” 25 Apr. 2016, 9:02 a.m. Tweet.

NSA Prism (NSA_PRISMbot). "Bria Bogan of El Vinnie, Alabama saved a file called http://habbo.zip on Google Drive." 22 Apr. 2016, 7:16 a.m. Tweet.

Pixel Sorter (pixelsorter). “@P_lucoli: hi.” 25 Apr. 2016, 2:25 p.m. Tweet.

Quilt Bot (a_quilt_bot).“æfluffy.” 7 Mar. 2015, 12:21 p.m. Tweet.

Restroom Genderator (RestroomGenderator). "heretical artists." 26 Apr. 2016, 4:59 a.m. Tweet.

SF QuakeBot (earthquakesSF). "A 1.7 magnitude earthquake occurred 5.59mi NNE of Milpitas, California. Details: http://eqbot.com/jjq Map." 23 Apr. 2016, 4:14 a.m. Tweet.

Stealth Mountain (stealthmountain). Stealth Mountain. “@BigBangCBS I think you mean 'sneak peek."' 8 Jan. 2014, 8:15 p.m. Tweet.

Two Headlines (TwoHeadlines). “T-Mobile wants his drug-test results made public.” 26 Apr. 2016, 9:08 a.m. Tweet.

Please Rap About (PleaseRapAbout). “@tomgalle whats wrong with selfies? Cause this girl satisfies Hulder folk and fairies LIKE BROKEN BLACK BERRIES http://pleaserapabout.com/QyUZVf7he." 28 Oct. 2015, 7:29 a.m. Tweet.

This Is Just To Say (JustToSayBot). "I have repressed the worlds that were in the lighter Forgive me They were impolite so snapped and so chaired." 25 Apr. 2016, 5:41 p.m. Tweet. 
Persona Studies 2016, 2 (1)

Žižek, Slavoj. Organs without Bodies: On Deleuze and Consequences. NY: Routledge Classics, 2012. Print.

\section{EDITOR'S NOTE:}

This submission has been peer reviewed. 